\title{
Storage of verbal associations is sufficient to activate the left medial temporal lobe
}

\author{
Andrew R. Mayes ${ }^{\mathrm{a}, *}$, Patricia A. Gooding ${ }^{\mathrm{b}}$, \\ Nicola M. Hunkin a , Julia A. Nunn ${ }^{\mathrm{a}}$, \\ Lloyd J. Gregory ${ }^{\mathrm{c}}$, Michael J. Brammer ${ }^{\mathrm{d}}$, \\ Edward T. Bullmore ${ }^{\mathrm{d}}$, Vincent Giampietro ${ }^{\mathrm{d}}$, \\ Rob Van Eijk ${ }^{\mathrm{b}}$, Amanda K. Nicholas ${ }^{\mathrm{a}}$ and \\ Steve C.R. Williams ${ }^{\mathrm{c}}$ \\ ${ }^{\mathrm{a}}$ Department of Clinical Neurology, University of \\ Sheffield, Sheffield S10 2JF, UK \\ ${ }^{\mathrm{b}}$ Department of Psychology, University of \\ Strathclyde, Glasgow G1 1XQ, UK \\ ${ }^{\mathrm{c}}$ Neuroimaging Unit \\ ${ }^{\mathrm{d}}$ Brain Image Analysis Unit, Institute of Psychiatry, \\ De Crespigny Park, London SE5 8AF, UK
}

Neuroimaging studies have shown that memory encoding activates the medial temporal lobe (MTL). Many believe that these activations are related to novelty but it remains unproven which is critical - novelty detection or the rich associative encoding it triggers. We examined MTL activation during verbal associative encoding using functional magnetic resonance imaging. First, associative encoding activated left posterior MTL more than single word encoding even though novelty detection was matched, indicating not only that associative encoding activates the MTL particularly strongly, but also that activation does not require novelty detection. Moreover, it remains to be convincingly shown that novelty detection alone does produce such activation. Second, repetitive associative encoding produced less MTL activation than initial associative encoding, indicating that priming of associative information reduces MTL activation. Third, re-encoding familiar associations in a well-established way had a minimal effect on both memory and MTL activation, indicating that MTL activation reflects storage of associations, not merely their initial representation.

Keywords: Hippocampus, medial temporal lobe, associative memory, encoding, priming

\section{Introduction}

Medial temporal lobe (MTL) damage is known to disrupt memory for episodic and factual associations [15]. There is also evidence, however, that MTL

${ }^{*}$ Corresponding author: Professor A.R. Mayes, Department of Clinical Neurology, N Floor, Royal Hallamshire Hospital, Sheffield S10 2JF, UK. Tel.: +44 (0)114 271 3431; Fax: +44 (0)114 276 0095; E-mail: A.Mayes@Sheffield.ac.uk. damage in humans disrupts attentional orienting towards information detected as novel when this is indicated by changes in autonomic responding or changes in event-related responses in EEG [14]. In animals, similar evidence indicates that behavioural orienting to certain kinds of novel information is disrupted by MTL lesions [13]. Parallel claims have been made with respect to the processes that underlie the MTL activation observed when subjects carry out encoding tasks in neuroimaging studies of memory. Thus, it has been suggested that the critical factor underlying MTL encoding-related activations is either associative encoding $[11,12]$ or novelty detection $[6,24]$, although both factors may be contributing. Another factor should also be considered. The repeated encoding of information as occurs in some priming studies may produce less MTL activation than the initial encoding of the information. Priming can be plausibly interpreted as memory that is indicated by an enhancement of the fluency of processing of repeated information, which by itself does not lead to the realization that the information is being remembered (for example, see [27]). In other words, enhanced fluency of processing (priming), which occurs in specific encoding situations, should reduce MTL activation compared to the similar encoding of novel information (see [18]).

Neuroimaging studies involving the encoding of novel information have, however, typically not attempted to control what is encoded, nor monitored whether novelty has been consciously detected and received enhanced attention. Furthermore, it has not been checked whether, if there has been attentional orienting to detected novelty, this has produced more elaborative encoding of the novel information. In such studies, high levels of novelty detection cannot be assumed in the absence of monitoring because subjects' attention may be largely focused on encoding the novel materials rather than determining whether they are novel or not. Even if novelty is detected in a comparison of the encoding of novel and familiar stimuli, MTL activation could be produced by one or more of several distinct processes. It could reflect: (a) the triggering of an orienting response; (b) the richer 
encoding given to novel information, or (c) the fact that re-encoding already studied (familiar) information in the same way as before produces less MTL activation. The third postulated process corresponds to priming, and fits with evidence that priming is associated with relative deactivation (see [18]). A priming deactivation of this kind could explain the effects found in encoding studies such as those of Dolan and Fletcher [6], which was used to argue that MTL activation during encoding reflects novelty detection. One can only conclude that the effects of novelty on the MTL are distinct from those produced by encoding information into memory and priming if a key condition is met. This is that the encoding of novel information can be shown to activate the MTL more than the encoding of similar, but already familiar information, solely because subjects detect and orient towards novelty. This condition is only met provided that the novel and familiar information is encoded in the same way, and with similar effects on memory (see [18]). Even if this is found, the MTL effect could nevertheless still reflect priming rather than novelty detection. If, however, it could be shown that novelty detection is not occurring, then an MTL priming effect has been demonstrated.

Lesion work results are consistent with two major suggestions about the memory functions of the MTL. The first suggestion is that the MTL is critical for putting information into memory (i.e., consolidation), but not for the representation of information at input when it is being encoded. This is because MTL damage does not seem to impair information processing when memory load is minimized (see [15]). The second suggestion is that the MTL seems to be more concerned with putting associative information into memory than it is with putting non-associative (item) information into memory. Taken together these two suggestions imply that the MTL is not needed to represent associations during study, but to consolidate the represented associations into memory. Support for the second suggestion is provided by evidence that selective hippocampal damage mainly impairs recognition for associations between different kinds of information that are likely to be represented in different neocortical regions (e.g., word-face), but not for recognition memory of single items such as words or faces $[16,25]$. In patients with large MTL lesions that include the MTL cortices, it has also been shown that recognition memory for associations between similar kinds of information (words) was more impaired than recognition memory for single items [17]. In other words, the MTL seems to be more concerned with pro- ducing memory for associations between either similar or dissimilar kinds of information than it is with producing memory for single items.

In agreement with these suggestions, neuroimaging studies recently showed that encoding of associations between both similar and dissimilar kinds of information activated the MTL more than did the encoding of single items $[11,12]$. Recent single trial functional magnetic resonance imaging (fMRI) studies have also found evidence that greater MTL activation was produced by the encoding of information that was subsequently remembered compared to the encoding of similar information that was not remembered later $[3,26]$, and the same thing was found in a study using an intra-subject correlational fMRI procedure [8]. Although these studies did not show a specific MTL role for associative memory consolidation, they suggested (although they did not prove) that the MTL mediates memory consolidation rather than merely representational processing at input.

In this study, we sought first to determine whether encoding of inter-word associations into memory produced more MTL activation than the encoding of single words when novelty was matched and novelty detection monitored across conditions. Examining verbal associative encoding was of particular interest because one reason why verbal encoding has often failed to activate the MTL is because it may frequently be non-associative [12]. We compared the novel associative encoding of three unrelated words (NAE), with the non-associative single item encoding of such words (SIE; see Table 1). The words in both conditions were novel in the experimental context, and were equally unrelated. However, convincing confirmation that any MTL effect related to associative encoding does not reflect novelty detection requires that novelty has been detected to a similar degree across conditions.

Our second aim was to determine whether repeatedly re-encoding the same triplet in the same way produced less MTL activation than making this kind of associative connection for the first time with novel word triplets. We therefore compared the NAE condition with an overlearnt associative encoding (OAE) condition. This comparison was intended to examine whether priming of verbal associative information produces deactivation of the MTL relative to the initial encoding of such associations with novel triplets. As indicated above, showing that any such effect reflects priming rather than novelty detection requires that minimal levels of novelty detection have occurred with the encoding of novel triplets. 
Table 1

Outline of the four verbal encoding tasks that were compared using fMRI

\begin{tabular}{|c|c|c|c|}
\hline Task & Material type & Requirement & Example \\
\hline $\begin{array}{l}\text { Novel associative } \\
\text { encoding (NAE) }\end{array}$ & Unrelated triplet & $\begin{array}{l}\text { Generate a sentence to link } \\
\text { the words. }\end{array}$ & $\begin{array}{c}\text { The large Gorilla } \\
\text { put the Cheese } \\
\text { on its Toe }\end{array}$ \\
\hline $\begin{array}{l}\text { Single item encoding } \\
\text { (SIE) }\end{array}$ & Unrelated triplet & $\begin{array}{l}\text { Using three simple sentences, } \\
\text { categorise each word using } \\
\text { the most specific category } \\
\text { applicable. }\end{array}$ & $\begin{array}{l}\text { Hammer is a tool } \\
\text { Coins are money } \\
\text { Wheat is a cereal }\end{array}$ \\
\hline $\begin{array}{l}\text { Familiar associative } \\
\text { encoding (FAE) }\end{array}$ & Related triplet & $\begin{array}{l}\text { Using three simple sentences, } \\
\text { categorise each word using } \\
\text { the most specific category } \\
\text { applicable to all three words. } \\
\text { The category should be modi- } \\
\text { fied with items two and three } \\
\text { if appropriate, as indicated in } \\
\text { the example. }\end{array}$ & $\begin{array}{l}\text { Orange is a fruit } \\
\text { Grapefruit is a citrus fruit } \\
\text { Apple is a fruit }\end{array}$ \\
\hline $\begin{array}{l}\text { Overlearnt associative } \\
\text { encoding (OAE) }\end{array}$ & Unrelated triplet & $\begin{array}{l}\text { Produce a sentence which } \\
\text { has been provided by } \\
\text { experimenter and learned } \\
\text { for one week prior to } \\
\text { scanning. }\end{array}$ & $\begin{array}{l}\text { viz. 'The Toddler hid the } \\
\text { Bag in the large } \\
\text { Greenhouse' }\end{array}$ \\
\hline
\end{tabular}

Our third aim was to determine whether encoding inter-word associations that had been encoded repeatedly on previous occasions outside the experimental context not only produced less MTL activation than encoding such associations for the first time, but also produced very little memory that these associations occurred in the study episode. If this happened, it would support the view that MTL activation reflects memory consolidation rather than merely the represention of associations during study. The behavioural effect of repetitive encoding on memory performance could not be explored with the OAE condition because the condition required ceiling performance before scanning began. We, therefore, compared the NAE condition with a familiar associative encoding condition (FAE; see Table 1) in which semantically related word triplets were only encoded once in the scanner. These words were, therefore, novel in the scanning encoding context, but the association between them was already well established outside this context and so was not novel.

In summary, novel associative encoding (NAE) of verbal material was compared with each of three conditions: single item encoding (SIE), overlearnt associative encoding (OAE) and familiar associative encoding (FAE). In order to check the effects on memory of encoding in the NAE, SIE, and FAE conditions, immediately after scanning was completed, subjects were given a recognition test for the specific triplets that they had been shown in the NAE, SIE and FAE conditions. The aims of the study were, therefore, to determine:

(a) whether verbal associative encoding processes elicit more MTL activation than do processes involved in encoding words non-associatively when stimulus novelty and novelty detection are both matched across conditions;

(b) whether the repeated encoding of the same verbal associative information produces less MTL activation than initial encoding even when there are minimal cross-condition differences in novelty detection (associative priming); and

(c) whether the MTL activating effect of novel associative encoding depends on memory for the inter-word associations being produced.

\section{Methods}

\subsection{Subjects}

Six healthy, right-handed male volunteers with a mean age of 23 years (range: 21 to 27 years). All subjects gave informed consent. The study was approved by the Bethlem and Maudsley NHS Trust Ethical Committee (Research).

\subsection{Design}

A within-subjects design was used with each subject contributing data for each of the three scan activation 
comparisons: NAE vs SIE; NAE vs OAE; and NAE vs FAE.

\subsection{Materials}

The word triplets used for the NAE and SIE conditions were prepared by re-combining semantically related concrete nouns as illustrated in Table 1, which also shows the specific triplet of unrelated words used for the OAE condition. The word triplets used for the FAE condition comprised related words all three of which fell within one semantic category as Table 1 illustrates. Use of words in the three different conditions was counterbalanced across subjects. For each subject, a single post-scan recognition test was prepared for the NAE, SIE, and FAE conditions. This test comprised several kinds of items in order to tap whether the subjects showed recognition memory for the studied word triplets or merely for studied words. Thus, some triplets from each condition were unchanged at test from how they had been during the scanning study episode (associative targets) whereas others were recombined such that the first, second, and third words of each test triplet were taken from different studied triplets used in the corresponding condition (associative foils). Associative recognition was tested in this way for the NAE and SIE conditions with targets and foils comprising unrelated triplet words whereas it was tested for the FAE condition using targets and foils comprising related triplet words that fell within one semantic category. In addition, a further set of triplets was prepared comprising words, none of which had been studied, but drawn from the same set as the studied triplets (item foils). For conditions NAE and SIE the foil triplets comprised unrelated words whereas for condition FAE they comprised related words. Use of these foils, made it possible to determine the level of subjects' recognition for individual studied words where inter-word associative memory was not involved.

\subsection{Procedures}

Whilst undergoing fMRI, subjects were presented visually with a series of single words. Each word was presented for three seconds by back-projection onto a translucent screen placed over the end of the scanner bore. After the third word, an asterisk appeared briefly to indicate the completion of a triplet. During each five-minute scan, two encoding tasks were alternated every 30 seconds so that for each 30 second period three triplets were presented, one every ten seconds. As each triplet was being presented, subjects were required to engage covertly in the appropriate encoding task. At the end of each 30 second period, subjects were briefly visually reminded of the next encoding task. Extensive practice was given to each of the subjects on the four tasks prior to scanning. Subjects were given the OAE triplet and the sentence with which they had to link the three unrelated words, one week before the scanning session. They were required to learn this sentence to the point where their production of the sentence was very fluent. Immediately prior to scanning, subjects were trained on the encoding that they would be asked to perform in the scanner for each of the four conditions. This was continued until each subject performed correctly and was confident that they understood what to do and that they could do it. The nature of the four encoding tasks is indicated in Table 1. In order to maximize any activational effects, subjects were trained to begin associative sentence production in the NAE and OAE conditions as soon as the first word was presented. In the FAE condition, associative encoding was ensured not only because all words in a triplet were related to a common semantic category through the production of three categorizing sentences, but also because subjects had to think of all three words together in order to identify the most specific category appropriate to them. The categorizing sentences were not novel encodings because subjects would have done such categorizing myriad times outside the experimental context. In the scanner, the order of the three comparisons was rotated across subjects.

Immediately after the end of the scanning session, subjects' recognition memory was tested outside the scanner using studied triplets, recombined triplets (comprising studied items displayed in new combinations), and new triplets (comprising novel items). Examples of studied and recombined triplets are shown in Fig. 1. Subjects were asked to identify only those triplets which were exactly the same as those they had seen in the scanner. For the FAE triplets, the recombination foils comprised items from the same semantic categories as were used during the study session. An approximate estimation of item recognition was made by measuring the tendency of subjects to make false alarms to recombination triplets to a greater extent than to completely new triplets. Associative recognition was estimated from the tendency of subjects to select as old correct triplets to a greater extent than recombination triplets. Memory was scored using signal detection theory. 


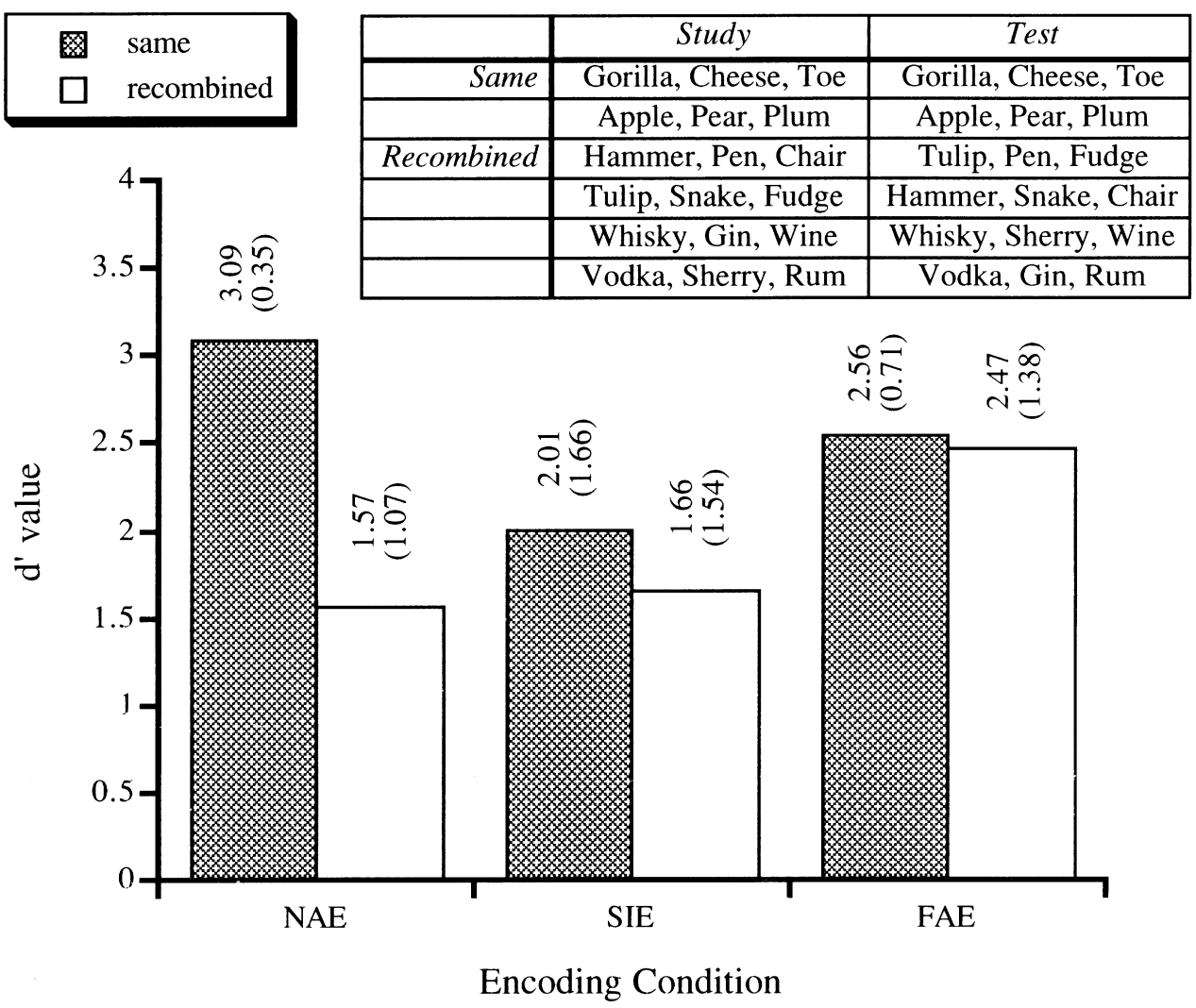

Fig. 1. Mean recognition performance for the six subjects included in the analysis expressed as mean d' is shown for same and recombined triplets in each of the three encoding conditions. The false alarm rate for the d' calculations was based on the proportion of incorrect selections of the completely novel word triplets. A d' value for associative recognition was calculated by subtracting the d' values for the recombined triplets from studied triplets for each of the three encoding conditions (d' NAE $=1.52$ [s.d. $=0.86$; d' SIE $=0.35$ [s.d. $=0.58$ ]; d' FAE $=0.09$ $[$ s.d. $=1.22]$. An ANOVA where type of association was a within subjects factor (novel, familiar, single) with linear contrast effects indicated that the difference scores were greater for the NAE condition than the other two $(F(1,10)=6.00, p<0.05)$, but did not differ between FAE and SIE conditions. In fact, associative recognition in these two latter conditions was not above chance levels $(F(1,5)=0.031, p=0.87$ and $F(1,5)=2.175, p=0.2$ respectively). An approximate estimate of non-associative word recognition is provided by the d' score for the recombined triplets.

Between each of three scans, subjects were asked to estimate the proportion of triplets in each condition they had encoded appropriately, but between scan debriefing of novelty detection was not attempted because the initial debriefing itself would have drawn attention to novelty in the later comparisons.

\subsection{Simulation study}

Instead, novelty detection during the four encoding tasks was examined on another occasion using 14 subjects, who were similar to those used in the scanning study. With these subjects, a simulation of the scanning procedure that included a recording of the noise of the scanner was carried out. Seven subjects were run in the NAE-SIE comparison and seven in the NAE-FAE comparison followed immediately by the NAE-OAE comparison. Immediately following the last comparison run, subjects were asked a series of questions which centrally included whether they had noticed that the words in each condition had been novel (to tap detection of item novelty within the study context) and whether they had detected that they were unrelated (associative novelty that was not confined to any specific context). It was stressed to the subjects that they were being asked whether they had detected that the words and the triplets were novel in these senses actually at the time that they were doing the encoding tasks in the scanning simulation.

\section{6. fMRI procedure}

Gradient recalled echoplanar MRI data were acquired using a GE Signa 1.5 Tesla system (General 
Electric) retrofitted with advanced NMR hardware using a standard head coil. $100 \mathrm{~T} 2 *$-weighted images depicting BOLD contrast [19] were acquired at each of 14 noncontiguous near-axial planes $(7 \mathrm{~mm}$ thick, $0.7 \mathrm{~mm}$ slice skip) parallel to the intercommissural (AC-PC) line; $\mathrm{TE}=40 \mathrm{~ms}, \mathrm{TR}=3 \mathrm{~s}$, flip angle $=90^{\circ}$, number of signal averages $=1$. Rigid body motion in 3D was estimated and corrected by realignment and regression [4]. Periodic change in MR signal intensity at the frequency of alternation between experimentally contrasted conditions was estimated by fitting a sinusoidal regression model to the motion-corrected fMRI time series observed at each voxel [5]. The standardised power at experimentally determined frequency was represented at each voxel in a parametric map. The observed time series was randomly permuted 10 times at each voxel, and standardised power was estimated after each permutation. Observed and permuted power maps were transformed into the standard space of Talairach and Tournoux [23], and smoothed by a 2D Gaussian filter with full width at half maximum $=7 \mathrm{~mm}$ [2]. The median observed power at each intracerebral voxel in standard space was tested against a critical value of the permutation distribution for median power ascertained from the permuted power maps. For a one-tailed test of size alpha, the critical value was the $100 \times(1-$ alpha $)$ th percentile value of the permutation distribution. Voxels for which observed median power exceeded this critical value were considered activated and coloured according to the timing of response. Activated voxels were superimposed on a grey scale EPI template image to form a generic brain activation map (GBAM) [2].

\section{Results}

The off-line simulation of all three comparisons indicated that novelty detection whilst performing all encoding tasks was minimal. This was unsurprising because subjects reported that effectively all their time had been spent performing the encoding task. In other work, we have found that this lack of aware novelty detection of both items and associations occurs in similar verbal encoding studies, but that subjects may still detect novelty when engaged in encoding of more complex and completely novel pictorial materials (see [18]).

\subsection{NAE vs SIE}

In the first fMRI comparison (NAE vs SIE), stimulus novelty was not only matched, but debriefing in the simulation study indicated that novelty detection was minimal and equivalent across conditions. NAE produced more posterior left MTL activation than SIE (see Fig. 2a and Table 2).

This effect was closely tied to memory because only in the NAE condition did subjects recognize significantly more studied word triplets than recombinations of studied words $(p<0.05)$. Associative recognition following SIE was at chance level although both the NAE and SIE conditions produced very similar and reasonable levels of word recognition as is shown in Fig. 1.

\subsection{NAE vs $O A E$}

In the comparison between the NAE and OAE conditions, NAE again produced more posterior left MTL activation (see Fig. 2b and Table 2). As novelty detection in the NAE condition was minimal in the simulation study, the difference between initial and repeated verbal associative encoding cannot be explained in terms of such detection.

\subsection{NAE vs FAE}

Finally, in the NAE vs FAE comparison, the NAE condition again produced more posterior left MTL activation and better associative memory performance than FAE. Associative recognition following FAE was actually at chance although single word recognition for the words in this condition was good as is shown in Fig. 1.

\subsection{Overlapping encoding activations}

As is shown in panel d of Fig. 2, when the relevant image slices from the three comparisons were superimposed on each other, the only overlapping sites were in the left posterior MTL. The co-ordinates of these overlapping activations are given in Table 2.

\section{Discussion}

Our results clearly show that the initial encoding of associations between words activates the posterior left MTL to a greater extent than either non-associative encoding of words, or associative encoding that has been carried out many times. It is important to note that the three comparisons showed activations that overlapped. This overlap occurred in a region that corresponds to the posterior MTL where most fMRI encoding-related 


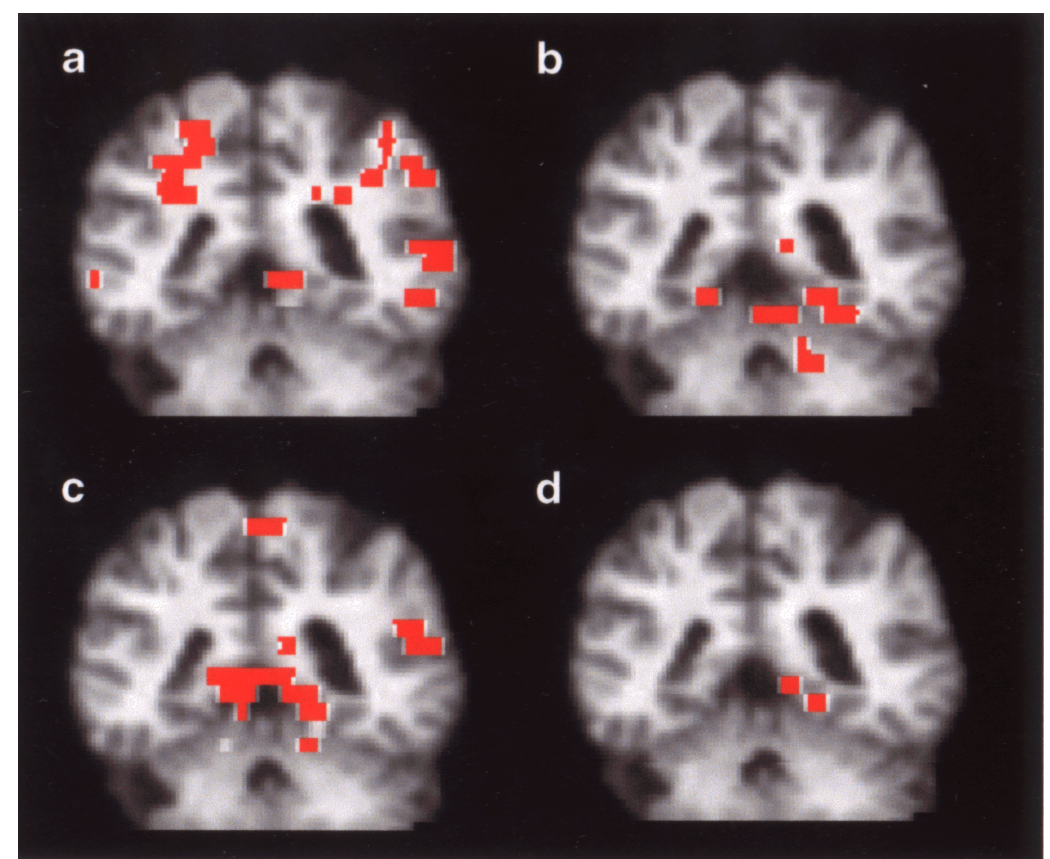

Fig. 2. Four activation maps superimposed on an SPGR image transformed into the standard stereotactic space of Talairach and Tournoux ${ }^{15}$. All four images are coronal sections at $y=-40 \mathrm{~mm}$. Left hemisphere of the brain corresponds to right side of image. Panels a, b and c show voxels activated ( $p<0.001$ by randomisation - see text) in the NAE condition relative to (a) SIE, (b) OAE and (c) FAE. Panel d shows only those voxels activated in the NAE condition in two or more of these three comparisons.

Table 2

Summary of left MTL activations in each of the three comparisons. Activations in 'bold' are the posterior MTL activations which contribute to those seen in Fig. 1.

\begin{tabular}{lcccc}
\hline Comparison & $\begin{array}{c}\text { Talairach } \\
\text { coordinates }\end{array}$ & $\begin{array}{c}\text { Maximum } \\
\text { FPQ }\end{array}$ & $\begin{array}{c}\text { Probability } \\
(\text { max FPQ) }\end{array}$ & $\begin{array}{c}\text { No. of voxels } \\
\text { activated }\end{array}$ \\
\hline NAE vs SIE & $\mathbf{- 9 , - 3 3 , - 2}$ & 1.6 & 0.000487 & 12 \\
& $\mathbf{- 6 , - 3 9 ,} \mathbf{4}$ & $\mathbf{1 . 7}$ & $\mathbf{0 . 0 0 0 1 5 5}$ & $\mathbf{7}$ \\
NAE vs OAE & $-17,-31,-2$ & 1.9 & 0.000005 & 30 \\
& $-32,-44,-2$ & 1.8 & 0.000005 & 31 \\
& $\mathbf{- 2 3 , - 3 9}-\mathbf{- 7}$ & $\mathbf{1 . 8}$ & $\mathbf{0 . 0 0 0 0 0 5}$ & $\mathbf{1 3}$ \\
NAE vs FAE & $\mathbf{- 3 5 , - 1 1 , - 1 3}$ & 1.7 & 0.000086 & 3 \\
\hline
\end{tabular}

activations have been found (see [21] for a review). It, therefore, seems likely that we have identified what is at least part of a single processing system, in a single set of neurons. This system probably creates verbal associative memory, because only the NAE condition produced associative memory; the other conditions were either not associative, or did not result in associative memory, and novelty detection was minimal in all conditions. The exact location of this associative memory system is beyond the current spatial resolution of fMRI, which makes it difficult to determine whether an activation involves the hippocampal region or the adjacent parahippocampal cortex. However, as hip- pocampal lesions may not disrupt inter-word association memory $[16,25]$ whereas large MTL lesions disrupt such associative memory more than word memory [22] creating inter-word association memories should primarily activate the MTL cortices rather than the hippocampus.

Since our study was completed, Henke et al. [12] have published a similar positron emission tomography (PET) study which also found that encoding associations between unrelated words activated the MTL more than did the non-associative encoding of words. There were several differences between the study of Henke and her colleagues and our study. They used 
abstract words organized into unrelated pairs and associative encoding simply involved judging whether the words 'fitted' well with each other or not. Although this associative encoding condition was somewhat unconstrained, it produced significantly more associative recognition memory than did the comparison conditions of encoding the words in each pair separately and either deeply or shallowly. The study also included an additional associative encoding condition in which encoded words had been pre-exposed although all of the encoded word pairs were novel prior to study in the PET scanner. Both the encoding conditions produced more MTL activation than did the non-associative encoding conditions. This means that associative verbal encoding produced more MTL activation than nonassociative verbal encoding even when the individually encoded words were novel in the context whereas the associatively encoded words were not. Taken together with our finding, the study of Henke and her colleagues strongly supports the view that verbal associative encoding produces more MTL activation than does non-associative verbal encoding under a variety of conditions. The activations reported by Henke and her colleagues were, however, more anterior in the MTL than those which we found. Whether this apparent difference reflects either processing differences between the studies or the possibility that fMRI may be less sensitive than PET to activations in the anterior MTL because of susceptibility artefact (see [21]) remains to be fully explored. It should be noted, however, that there is a tendency of workers to exaggerate the spatial resolution with which activations can be identified so the MTL activations found in the two studies may not be meaningfully different from each other.

A related and further important finding of the current study is that the re-encoding of associative information in the same way that it has been encoded many times previously outside the scanning situation not only reduces the level of MTL activation, but also produces insignificant levels of memory about which associations occurred in the study episode. This suggests two points of interest. First, repeating the same encoding operation on repeated inputs diminishes the beneficial effects on memory, which has not previously been demonstrated. Other work indicates that provided encoding operations have not been performed previously they produce clearcut effects on memory even when the input is repeated (see [18]). Second, the effect provides strong support for the view expressed above that MTL activation depends critically on the produc- tion of associative memories rather than merely on the representation of verbal associations at encoding.

Novelty detection was not required to produce the MTL activations reported here so our results are similar to those of two recent single trial fMRI studies $[3,26]$ and the PET study of Henke et al. [12] in that all four studies found that encoding information into memory activated the MTL when item and associative novelty were matched across conditions. Such matching makes it improbable that novelty detection could have been contributing to the effect, although a direct demonstration that this was not so would still be desirable. This is what we attempted to do in this study using the simulation procedure. Future work must determine whether item and/or associative novelty detection alone activates the MTL or whether it only does so when it triggers associative encoding and memory. If novelty detection alone is sufficient, it will be critical to identify whether it activates regions distinct from those involved in associative memory (see [18] for a discussion of this issue).

The effect found in the NAE vs OAE comparison is likely to reflect a priming-like phenomenon in which repeated performance of an encoding operation produced relatively less MTL activation. The MTL was probably the brain region where the primed verbal associations were represented, as several neuroimaging studies of priming have suggested that relative deactivation occurs in brain regions where the primed information is likely to be represented [27]. Priming often involves the retrieval of a stimulus in the same way that it has been encoded so that retrieval and reencoding involve overlapping processes. Consistent with our finding, making indoor/outdoor judgements repeatedly with the same two pictures was found to produce less MTL activation than making such judgements for the first time with novel pictures [9]. Unlike in our comparison, novelty detection was not examined as a possible MTL activating factor in the Gabrieli et al. study [9]. However, it has since been shown that encoding similar pictures into memory, using the same encoding task, activated the MTL even when novelty was matched across conditions so that novelty detection and its consequences would probably not have been contributing to the MTL activation produced by putting information about the pictures into memory [3]. It nevertheless remains possible that detecting pictorial novelty does activate the MTL. Furthermore, neither of these picture encoding studies $[3,9]$ controlled what was encoded and, consequently, it was not demonstrated that subjects were using associative 
memory. Our study is, therefore, the first to show directly that priming of associations involves the MTL. This finding is consistent with the view that the MTL initially stores associative information, as this would explain why associative priming causes deactivation of this region.

The priming-like phenomenon elicited by the NAE vs OAE comparison has important implications. The predominant view is that the MTL is solely concerned with aware fact and episode memory (declarative memory), and is not involved with unaware memory processes, such as the fluency enhancement processes that probably underlie priming [22]. The phenomenon we observed is inconsistent with this view because it suggests that the MTL is involved with the enhanced fluency that underlies priming as well as with the encoding of associations so as to produce aware memory (recognition). Our finding is, however, consistent with the results of a meta-analysis [10] which found that amnesic patients, including those with MTL damage, are impaired at novel item and associative information priming. This meta-analysis also implies that our results do not reflect psychological processes that are incidental to and not necessary for priming because it indicates that the MTL needs to be intact in order for associative priming to occur at normal levels. Our results are also concordant with those of the great majority of neuroimaging studies of non-associative priming in finding a deactivation in the brain region where the primed information is probably represented [20]. This pattern is consistent with single unit recording evidence that neuronal activity is often reduced in brain regions likely to be involved in storing information when that information is repeated [27]. Both our results and, slightly less strongly, those of Gabrieli and his colleagues suggest that the MTL shows reduced activation when verbal associations and pictorial information respectively are primed. In both cases, however, priming must have been accompanied by some recognition and cued recall (aware memory). This is unlikely to have confounded the effects of priming because these forms of aware memory retrieval often fail to influence activity of the MTL. However, the only way to avoid this potential confound completely will be to ensure that there is no aware memory for associatively primed items in future studies.

Two published neuroimaging studies of priming which have reported MTL priming effects need to be discussed, however, because they claimed to eliminate aware memory for studied materials $[1,7]$. This was achieved by presenting stimuli subliminally so that aware memory was later found to be at chance. Elliot and Dolan [7] reported that, following subliminal exposure to Japanese ideograms, subjects showed more activation in the right parahippocampal/fusiform cortex region when making judgements about objectively novel ideograms than when making similar judgements about ideograms which had previously been subliminally exposed. However, Beauregard et al. [1] reported more activation in the right anterior MTL region when subjects made semantic category decisions about words that had previously been subliminally preexposed than when making similar judgements about objectively novel words. Three comments should be made about these studies. First, it is not clear whether they show that priming of associations activates the MTL. If they do not, they may involve priming for kinds of information that are unaffected by MTL lesions [20] in which case the MTL effects must reflect a different and unknown process. Second, although the tasks are broadly similar in the comparisons being made, they involved distinct MTL activations. In the Elliot and Dolan study, MTL activation was posterior $(y=-38)$ whereas in the Beauregard study it was anterior $(y=-9)$. Third and most important, the MTL effects were in opposite directions, which indicates that replication is important for both studies, but particularly that of Beauregard et al. where the direction of effect was atypical for a neuroimaging study of priming.

In conclusion, the results of this study demonstrate that verbal associative encoding can elicit left MTL activation, but only when the associative processing is carried out for the first time so that processing results in episodic memory for the association. The activation is independent of novelty detection, although it remains to be seen whether novelty detection independently elicits MTL activation. Finally, the results of this study suggest that the MTL shows reduced activation when verbal associations are primed.

\section{Acknowledgements}

This study was supported by the Wellcome Trust (Grant number 037666). We are grateful to Daniela Montaldi for helpful discussions.

\section{References}

[1] M. Beauregard, D. Gold, A.C. Evans and H. Chertkow, A role for the hippocampal formation in implicit memory: a 3-D PET study, Neuroreport 9 (1998), 1867-1873. 
[2] M.J. Brammer, E.T. Bullmore, A. Simmons, S.C.R. Williams, P.M. Grasby, R.J. Howard, P.W. Woodruff and S. RabeHesketh, Generic brain activation mapping in functional magnetic resonance imaging: a nonparametric approach, Magnetic Resonance Imaging 15 (1997), 763-770.

[3] J.B. Brewer, Z. Zhao, G.H. Glover and J.D.E. Gabrieli, Making memories: brain activity that predicts whether visual experiences will be remembered or forgotten, Science $\mathbf{2 8 1}$ (1998), 1185-1187.

[4] E.T. Bullmore, M.J. Brammer, S. Rabe-Hesketh, V.A. Curtis, R.G. Morris, S.C.R. Williams, T. Sharma and P.K. McGuire, Methods for diagnosis and treatment of stimulus-correlated motion in generic brain activation studies using fMRI, Human Brain Mapping 7 (1999), 38-48.

[5] E.T. Bullmore, M.J. Brammer, S.C.R. Williams, S. RabeHesketh, N. Janot, A. David, J. Mellers, R. Howard and P. Sham, Statistical methods of estimation and inference for functional MR image analysis, Magnetic Resonance in Medicine 35 (1996), 261-277.

[6] R.J. Dolan and P.C. Fletcher, Dissociating prefrontal and hippocampal function in episodic memory, Nature 388 (1997), $582-585$.

[7] R. Elliot and R.J. Dolan, Neural response during preference and memory judgments for subliminally presented stimuli: A functional neuroimaging study, Journal of Neuroscience 18 (1998), 4697-4704.

[8] G.Fernandez, H. Weyerts, M. Schrader-Bolsche, I. Tendolkar, H.G.O.M. Smid, C. Tempelmann, H. Hinrichs, H. Scheich, C.E. Elger, G.R. Mangun and H-J. Heinze, Successful verbal encoding into episodic memory engages the posterior hippocampus: A parametrically analyzed functional magnetic resonance imaging study, Journal of Neuroscience 18 (1998), 1841-1847.

[9] J.D.E. Gabrieli, J.B. Brewer, J.E. Desmond and G.H. Glover, Separate neural bases of two fundamental memory processes in the human medial temporal lobe, Science 276 (1997), 264 266.

[10] P.A. Gooding, A.R. Mayes and R. Van Eijk, A meta-analysis of indirect memory tests for novel material in organic amnesics, Neuropsychologia (in press).

[11] K. Henke, A. Buck, B. Weber and H.G. Wieser, Human hippocampus establishes associations in memory, Hippocampus 7 (1997), 249-256.

[12] K. Henke, B. Weber, S. Kneifel, H.G. Wieser and A. Buck, Human hippocampus associates information in memory, Proceedings of the National Academy of Sciences USA 96 (1999), 5884-5889.
[13] R.C. Honey, A.Watt and M. Good, Hippocampal lesions disrupt an associative-mismatch process, Journal of Neuroscience 18 (1998), 2226-2230.

[14] R.J. Knight, Contribution of human hippocampal region to novelty detection, Nature 383 (1996), 256-259.

[15] A.R. Mayes and J.J. Downes, What do theories of the functional deficit(s) underlying amnesia have to explain?, Memory 5 (1997), 3-36.

[16] A.R. Mayes, R. van Eijk, P.A. Gooding, C.L. Isaac and J.S. Holdstock, What are the functional deficits produced by hippocampal and perirhinal cortex lesions?, Behavioral and Brain Sciences 22 (1999), 36-37.

[17] A.R. Mayes, C. MacDonald, L. Donlan, J. Pears and P.R. Meudell, Amnesics have a disproportionately severe memory deficit for interactive context, Quarterly Journal of Experimental Psychology 45A (1992), 265-297.

[18] A.R. Mayes and D. Montaldi, The neuroimaging of long-term memory encoding processes, Memory 7 (in press).

[19] S. Ogawa, T.M. Lee, A.R. Kay and D.W. Tank, Brain magnetic resonance imaging with contrast dependent on blood oxygenation, Proceedings of the National Academy of Sciences USA 87 (1990), 9868-9872.

[20] D.L. Schacter and R.L. Buckner, On the relations among priming, conscious recollection, and intentional retrieval: Evidence from neuroimaging research, Neurobiology of Learning and Memory 70 (1998), 284-303.

[21] D.L. Schacter and A.D. Wagner, Medial Temporal Lobe activations in fMRI and PET studies of encoding and retrieval, Hippocampus 9 (1999), 7-24.

[22] L.R. Squire and B.J. Knowlton, Memory, hippocampus and brain systems, in: The Cognitive Neurosciences, M. Gazzaniga, ed., MIT Press, Cambridge, Mass, 1995.

[23] J. Talairach and P. Tournoux, A Coplanar Atlas of the Human Brain, Thieme Verlag, Stuttgart, 1988.

[24] E. Tulving, H.J. Markowitsch, F.I.M. Craik, R. Habib and S. Houle, Novelty and familiarity activations in PET studies of encoding and retrieval, Cerebral Cortex 6 (1996), 71-79.

[25] F. Vargha-Khadem, D.G. Gadian, K.E.Watkins, A. Connelly, W. Van Paesschen and M. Mishkin, Differential effects of early hippocampal pathology on episodic and semantic memory, Science 277 (1997), 376-380.

[26] A.D. Wagner, D.L. Schacter, M. Rotte, M. Koutstaal, A. Maril, A.M. Dale and R.L. Buckner, Building memories: Remembering and forgetting of verbal experiences as predicted by brain activity, Science 281 (1998), 1188-1191.

[27] C.L. Wiggs and A. Martin, Properties and mechanisms of perceptual priming, Current Opinion in Neurobiology 8 (1998), 227-233. 


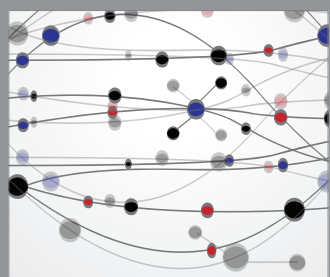

The Scientific World Journal
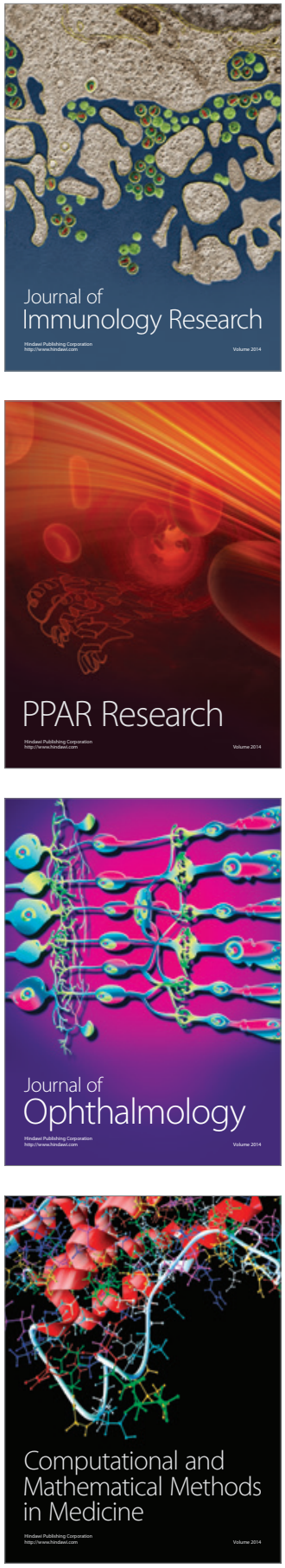

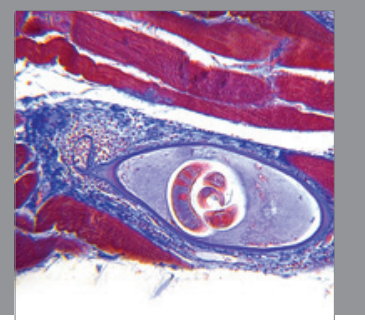

Gastroenterology

Research and Practice
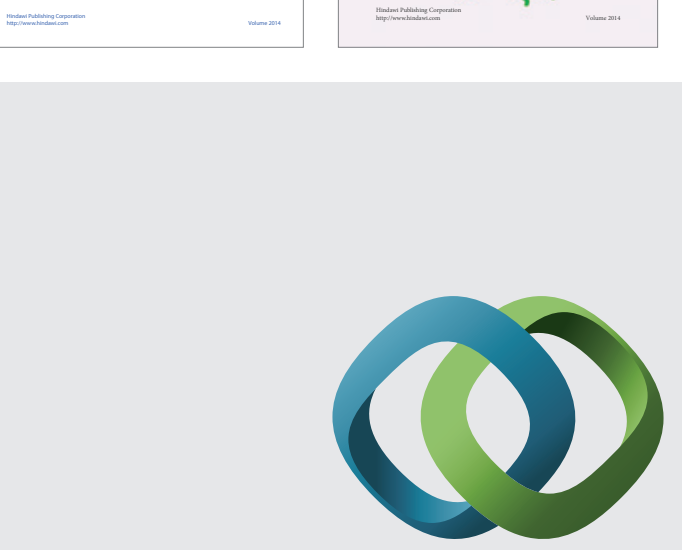

\section{Hindawi}

Submit your manuscripts at

http://www.hindawi.com
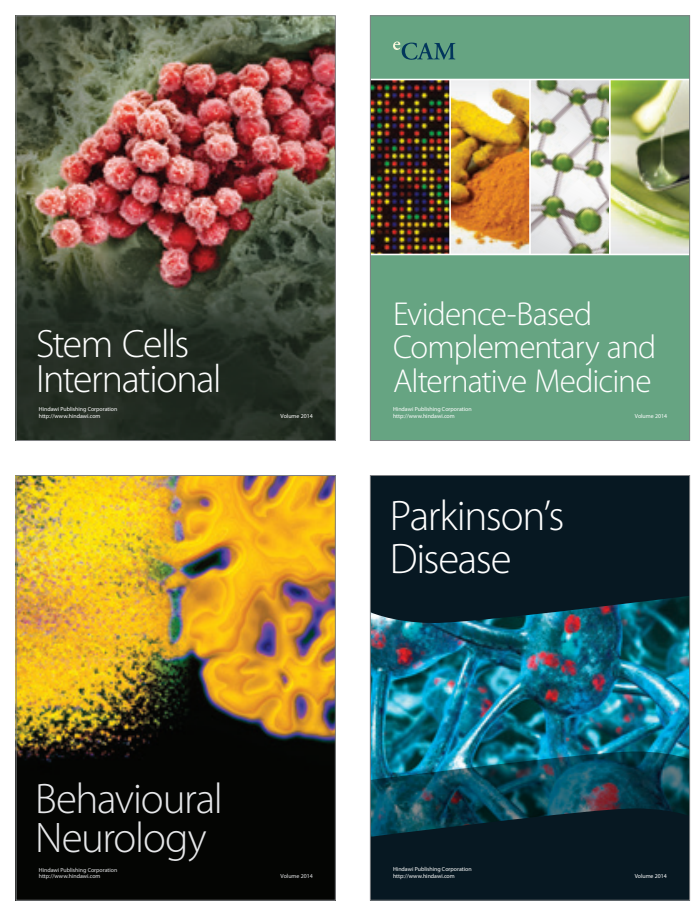

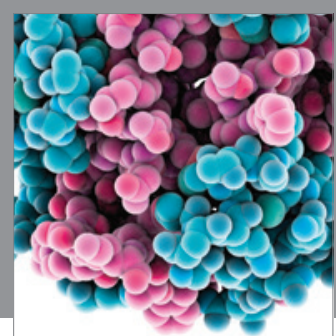

Journal of
Diabetes Research

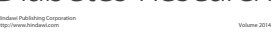

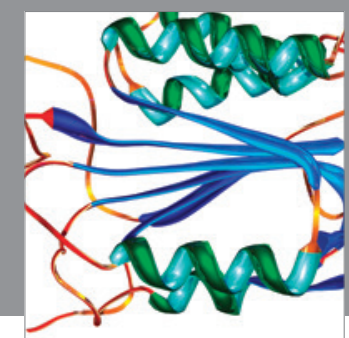

Disease Markers
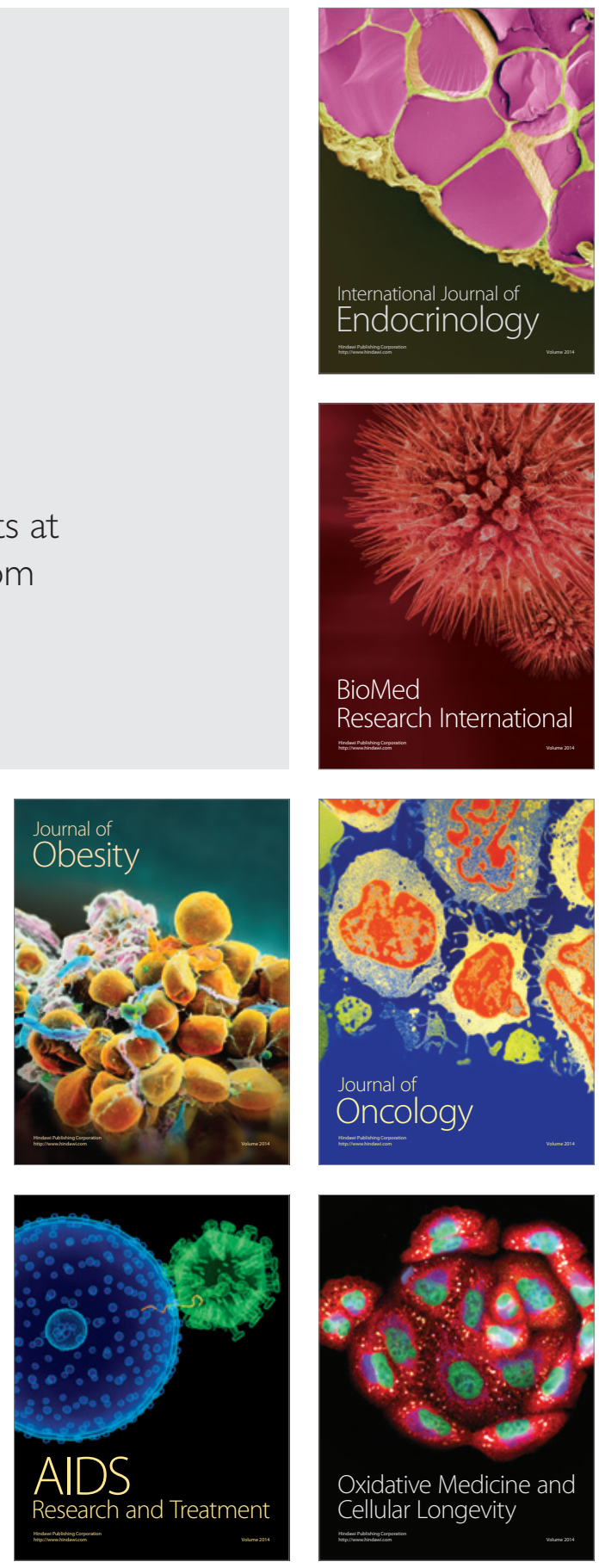